\title{
MODELING AND SIMULATION OF RECYCLED CARBON FIBER REINFORCED COMPOSITES WITH VARYING FIBER LENGTHS
}

\author{
Tobias Georg Lang ${ }^{1 *}$, Mir Mohammad Badrul Hasan ${ }^{1}$, Thomas Gereke ${ }^{1}$, \\ Anwar Abdkader ${ }^{1}$, Chokri Cherif ${ }^{1}$ \\ ${ }^{1}$ Technische Universität Dresden, Faculty of Mechanical Science and Engineering, \\ Institute of Textile Machinery and High Performance Material Technology (ITM), \\ 01062 Dresden, Germany \\ * tobias_georg.lang@tu-dresden.de
}

Due to the current trend towards lightweight design across diverse disciplines, the usage of composites such as carbon fiber reinforced polymers has increased enormously in the last decade. With the rising usage of carbon fibers, topics like repurposing of fibers are gaining more importance. One current approach is the production of hybrid yarns from recycled staple carbon fibers and thermoplastic fibers. During each processing step from fiber to hybrid yarn, the fibers are partially damaged [1]. The resulting broad fiber length distribution considerably affects the mechanical properties of the composite. Therefore, the influence of the different fiber lengths on the fiber structures and composite properties should be investigated thoroughly.

This work proposes a micro-scale modelling method for predicting the strength of composites of hybrid yarns made of carbon staple fibers, while paying a special attention to the influence of the fiber length. To consider their irregular nature, a method to generate stochastic fiber unit-cell models is established. The input parameters (e. g. length, cross section, orientation, waviness, tensile strength) are specified using stochastic data. By simulating the compaction of the generated fibers, the composite manufacturing process is considered. Finally, the compacted fibers are coupled within a matrix using kinematic constraints in the model. Along the fiber axis, a bond-slip model based on fiber-pullout-tests is applied [2]. The composite model is validated by comparing virtual mechanical tests with experimental test results.

\section{Acknowledgement}

The authors gratefully acknowledge the financial support of the German Research Foundation (Deutsche Forschungsgemeinschaft, DFG) in scope of the project CH 174/46-1.

\section{References}

[1] M. F. Khurshid, A. Abdkader and C. Cherif (2020) Processing of waste carbon and polyamide fibres for high-performance thermoplastic composites: influence of carding parameters on fibre orientation, fibre length and sliver cohesion force, The Journal of The Textile Institute, 111:9, 1277-1287, DOI: 10.1080/00405000.2019.1690918

[2] M. Hengstermann, M. M. B. Hasan, C. Scheffler, A. Abdkader, C. Cherif (2019) Development of a new hybrid yarn construction from recycled carbon fibres for highperformance composites. Part III: Influence of sizing on textile processing and composite properties. Journal of Thermoplastic Composite Materials. May 2019. DOI: $10.1177 / 0892705719847240$ 